\title{
Colon Cancer Perforation Presenting as a Strangulating Ventral Hernia
}

\author{
Pavlos Lampropoulos Emmanouil Liarmakopoulos \\ Charalambos Markakis Mavroudis Voultsos \\ Tammy Rosen Marinou Athanasios Marinis \\ Andreas Hadjimarkou Spyros Rizos \\ First Department of Surgery, 'Tzaneion' General Hospital, Piraeus, Greece
}

\section{Key Words}

Colon cancer · Unusual presentation of cancer · Perforation

\begin{abstract}
Colorectal cancer remains the second leading cause of death from malignant disease. Despite improvements in the treatment modalities offered to patients, more than half of the operated patients die from the disease. The most common presenting symptoms of colonic carcinoma are changes in bowel habits, bleeding, abdominal pain, abdominal mass, stools mixed with mucus or not, weight loss, anorexia, and other characteristics related to metastasis. Here, the case of a 74-year-old female patient with colon cancer perforation presenting as a strangulating ventral hernia and a mini-review of the current literature are presented.
\end{abstract}

\section{Introduction}

The primary goal in colon cancer patients is to cure the disease. Complete oncological surgical resection whenever possible is the cornerstone of treatment. In cases of locally advanced tumors with invasion of adjacent structures (T4), en bloc resection of all involved tissues is essential so as not to increase local failure and decrease survival. Adjuvant chemotherapy is used in order to eliminate any possibility of developing metastasis from cancer cell dissemination. In cases of perforated colon cancer, a dismal prognosis is anticipated due to the burden of the septic episode, in addition to the hazardous effect from the cancer. We present a case of perforated sigmoidal cancer with abscess formation to the ventral abdominal wall, which was, however, first misdiagnosed as strangulating ventral hernia. 


\section{Case Report}

A 74-year-old female patient was admitted to our surgical department complaining of a painful palpable mass of the hypogastrium and high fever, which developed over a period of a few hours. The patient reported changes in bowel habits, anorexia and weight loss during the last 2 months prior to admission. Physical examination revealed a temperature of $38^{\circ} \mathrm{C}$ and a palpable, protruding, and erythematous mass over a midline lower abdominal wall incision. Her past medical history was unremarkable except for an appendectomy 40 years earlier through the aforementioned incision. Routine laboratory data on admission showed leukocytosis (white blood cells $16,550 / \mu \mathrm{l}$, polymorphonuclears $77.9 \%$ ). Electrocardiographic recordings and chest radiographs were normal.

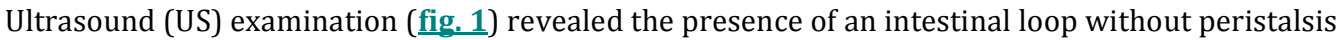
within the abdominal mass, and the diagnosis of a strangulated ventral hernia was set. The patient was scheduled for emergency surgery. Intraoperative findings (fig. 2 , fig. 3 ) demonstrated a large subcutaneous abscess and a perforated sigmoidal cancer adherent to the abdominal wall, invading it along with the urinary bladder. The surgical team decided that the sigmoidal cancer was operable and proceeded with an oncological surgical operation. The abscess was drained, and an en bloc resection of the invaded abdominal wall and urinary bladder was carried out, followed by a Hartmann procedure (resection of the sigmoid colon with a diverting colostomy and rectal stump closure).

Histopathology of the specimens revealed that none of the 15 lymph nodes harvested was invaded by the tumor. Immunohistochemistry showed positive reactivity to Ki67 and p53 in 90 and 80\% of the neoplastic nuclei, respectively. In addition, there was no reactivity for Bcl2, and identification of EGFR with Kit Dako EGFR pharm DX was negative. According to TNM classification, the patient was staged as T4N0M0 (stage IIB).

In the immediate postoperative period, the patient developed deep vein thrombosis of the left femoral and left common iliac veins and was treated accordingly. Computed tomography of the abdomen and thorax showed no pathology related to the malignancy other than the thrombosis. Following an oncological consultation, the patient was scheduled for adjuvant chemotherapy soon after recovering from the thrombotic episode. Written informed consent was obtained from the patient for publication of this case report and any accompanying images. A copy of the written consent is available for review by the Editor-in-Chief of this journal.

\section{Discussion}

Screening protocols for colon cancer have failed so far to recruit all patients at risk, mostly due to low socioeconomic conditions and poor education. Therefore, it is not uncommon for colon cancer patients to present with perforation, which might be free, contained or result in a fistula formation. Free perforation leads to secondary peritonitis, while contained or fistulous perforation has a variable outcome. Surgical options for perforated colon cancer include diverting colostomies, partial or subtotal colectomies in a single-stage operation or multiple-stages procedures, and various interventional endoscopic techniques [1].

Many institutions have reported outcomes following surgery for perforation of the colon. In a retrospective study of 129 patients, Tan et al. [2] showed that surgery after colonic perforation is associated with higher morbidity and mortality rates, while short-term outcome is associated with the severity of peritonitis and ASA score. In another retrospective study including 45 patients with perforated colorectal cancer [3], the same authors showed similar results and stated that long-term outcome in these patients is determined by cancer stage.

There are many studies in the literature reporting on unusual presentations of colonic cancer, including incarcerated inguinal hernia [4], gluteal abscess [5], and 
necrotizing fascitis of the thigh [6]. In our case, the sigmoid was adherent to the abdominal wall due to adhesion formation from the previous operation. The adherent part of the sigmoid contained the tumor. Soon after the tumor mass had ruptured, a fistulous tract formed between the bowel and the abdominal wall. This led to the subcutaneous accumulation of fecal content and the abscess formation. US examination misled the surgical team to diagnose an incarcerated ventral hernia.

Regardless of the clinical manifestations of colon cancer, the same oncological principles apply to all patients. Even in preoperatively undiagnosed colon cancer cases, surgeons must exhaust all possible means to perform a sound oncological operation. In our case, the surgical team managed to resect the invaded abdominal wall and the invaded part of the urinary bladder, and subsequently performed a Hartmann procedure. An issue of importance is the fact that our patient had a contained perforation, which has a more favorable outcome than a free perforation. In a retrospective study [7], patients with free perforated colon cancer had a 5-year disease-free survival of $15 \%$, while patients with a contained perforation had a disease-free survival of $53 \%(\mathrm{p}<0.001)$. Furthermore, free perforated colon cancer patients had a postoperative mortality rate of $19 \%$ compared with $0 \%$ in contained perforated patients.

\section{Conclusion}

It is quite difficult to recognize a perforated sigmoid tumor resulting in an abdominal wall abscess preoperatively. The abscess formation may further be misdiagnosed as a ventral hernia, especially if located over a previous abdominal incision. A high level of suspicion is required in order to make a correct diagnosis of perforated colon cancer to the abdominal wall, especially in older patients. Regardless of the clinical manifestation of bowel cancer, the appropriate surgical therapy is warranted.

\section{Disclosure Statement}

The authors declare that they have no competing interests. 


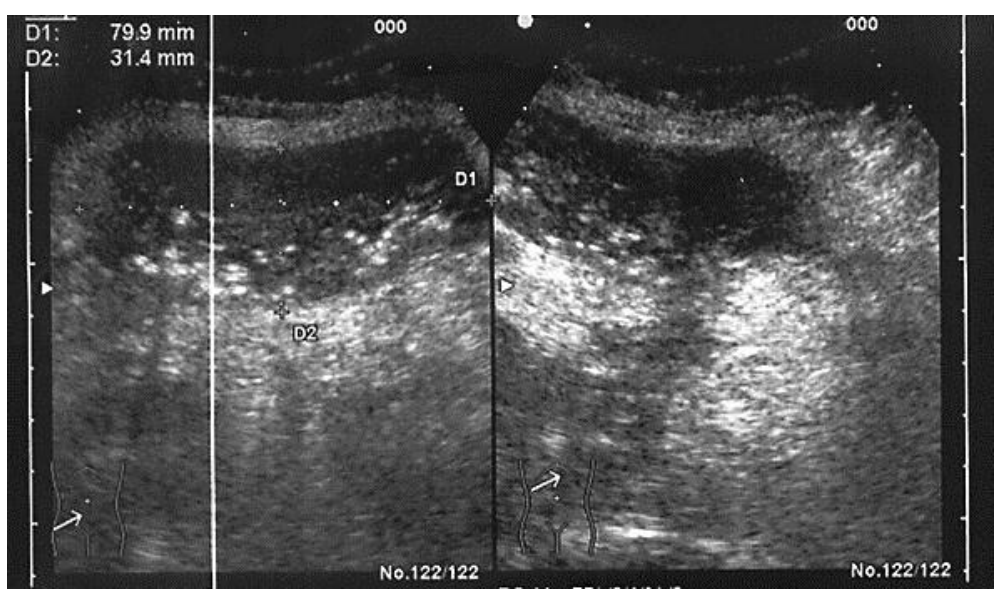

Fig. 1. US examination demonstrating an intestinal loop with no peristalsis.

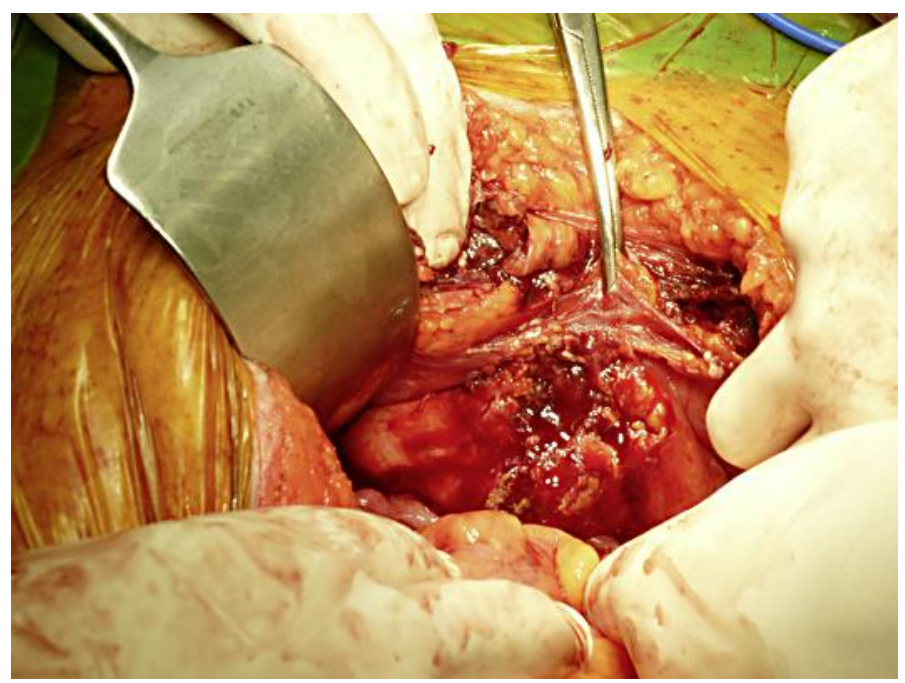

Fig. 2. Intraoperative photograph demonstrating colon perforation. 


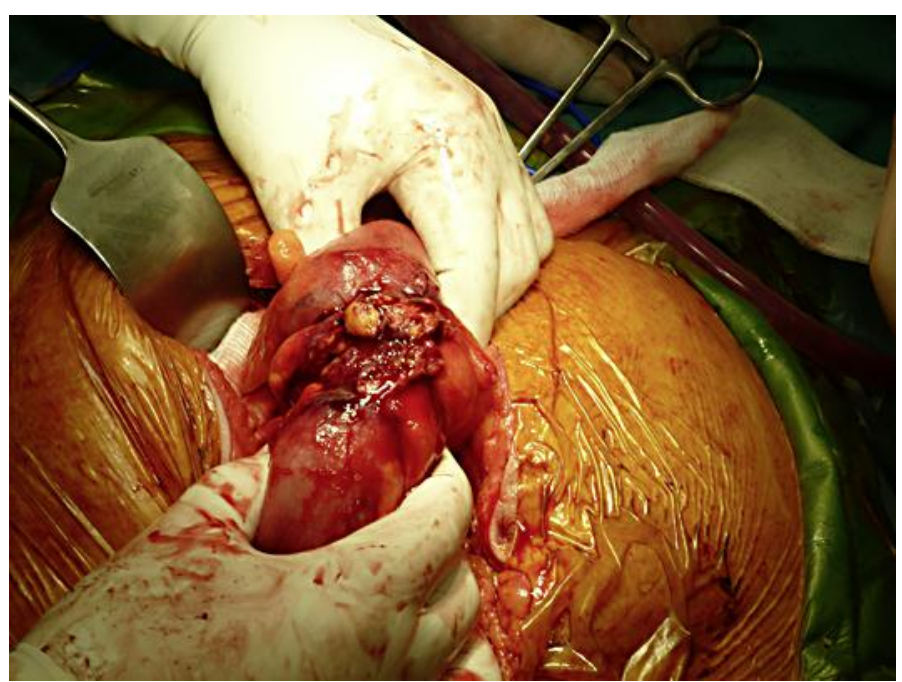

Fig. 3. Intraoperative photograph demonstrating colon perforation.

\section{References}

1 Schwenter F, Morel P, Gervaz P: Management of obstructive and perforated colorectal cancer. Expert Rev Anticancer Ther 2010;10:1613-1619.

2 Tan KK, Hong CC, Zhang J, Liu JZ, Sim R: Predictors of outcome following surgery in colonic perforation: an institution's experience over 6 years. J Gastro intest Surg 2011;15:277-284.

-3 Tan KK, Hong CC, Zhang J, Liu JZ, Sim R: Surgery for perforated colorectal malignancy in an Asian population: an institution's experience over 5 years. Int J Colorectal Dis 2010;25:989-995.

-4 Ruiz-Tovar J, Ripalda E, Beni R, Nistal J, Monroy C, Carda P: Carcinoma of the sigmoid colon in an incarcerated inguinal hernia. Can J Surg 2009;52:E31-E32.

-5 Mohandas SK, Mazarello F, Bisset R: Right gluteal abscess: an unusual presentation of perforated caecal adenocarcinoma. J Gastrointest Cancer 2010;41:285-287.

-6 Takakura Y, Ikeda S, Yoshimitsu M, Hinoi T, Sumitani D, Takeda H, Kawaguchi Y, Shimomura M, Tokunaga M, Okajima M, Ohdan H: Retroperitoneal abscess complicated with necrotizing fasciitis of the thigh in a patient with sigmoid colon cancer. World J Surg Oncol 2009; 7:74.

-7 Zielinski MD, Merchea A, Heller SF, You YN: Emergency management of perforated colon cancers: how aggressive should we be? J Gastrointest Surg 2011;15:2232-2238. 